\title{
Diversity Functions of Puf Proteins in Protozoan Species
}

\section{Liang $\mathrm{X}^{*}$}

Department of Entomology, Pennsylvania State University, USA

*Corresponding author: Xiaoying Liang, Department of Entomology, Pennsylvania State

University, University Park, PA 16802, USA, Email: xzl112@psu.edu

\section{Editorial \\ Volume 1 Issue 2}

Received Date: July 16, 2018

Published Date: July 20, 2018

\section{Editorial}

In all eukaryotic cells, the regulation of gene expression is accomplished at both transcriptional and translational levels. At the translational level, control of gene expression is often mediated by cis-acting elements in the 3' untranslated region (UTR) of the mRNA. This regulation is achieved through a network of RNA binding proteins (RBPs) that have great effect on stability, localization and translatability of their target mRNA. The Puf family RBPs named after Pumilio (Pum) protein in Drosophila melanogaster and fem-3 binding factor (FBF) protein in Caenorhabditis elegans [1,2], represent a highly conserved group of RBPs found in many organisms including animals, plants, fungi and protists [3-5].

So far, only a few Puf protein members have been identified in protozoan species. Two Puf RBPs (PfPuf1 and PfPuf2) of P. falciparum have been identified in 2002 [6]. PfPuf1 has a central Puf domain, whereas PfPuf2 has Cterminal Puf domain. Both of them are preferentially expressed in the gametocyte stages [6,7]. Disruption of PfPuf1 showed no noticeable phenotypic changes in the asexual stage of the parasites, but in the sexual stage, gametocytes showed growth defect after stage III [8]. Interestingly, whereas most Puf binding elements (PBEs) are localized in the $3^{\prime}$ UTR or $3^{\prime}$ UTR proximal coding region of target mRNAs, PfPuf2 has been found to repress Pfs 25 by binding to the PBE in the $5^{\prime}$ UTR of pfs 25 to mediate translation repression, suggesting that Puf proteins are versatile translation regulators $[9,10]$.

Most recently, another Puf protein, PfPuf3 has been characterized in P. falciparum. This protein is a nucleolar protein which participated in ribosomal biogenesis in malaria parasites [11]. In the rodent malaria parasite P. berghei, targeted deletion of Puf2 did not have any effect on asexual stages [12]. However, PbPuf2 knockout sporozoites showed apparent morphological changes and defects in motility, cell traversal and infection when compared with wild type parasites [13]. In contrast to
PbPuf2 knockout parasites, Puf2 knockout sporozoites in P. yoelii did not show apparent defect in host infection at first, but progressively become non-infectious [14]. In Toxoplasma gondii, only two Puf RBPS (TgPuf1 and TgPuf2) have been found and TgPuf1 protein exhibits different expression levels in bradyzoites and tachyzoites. RNA Electrophoretic Mobility Shift Assay (EMSA) showed that TgPuf1 has conserved RNA binding activity and specificity, suggesting that TgPuf1 may regulate mRNA translation during transition from bradyzoites to tachyzoites [15]. The first nucleolar Puf identified in 25 protozoan species is T. brucei Puf7. Deletion of TbPuf7 inhibited ribosomal RNA processing, resulting in retarded parasite growth [16]. All these data demonstrated that Puf RBPs in protozoan species have important functions.

\section{References}

1. Cui L, Fan Q, Li J (2002) The malaria parasite Plasmodium falciparum encodes members of the Puf RNA-binding protein family with conserved RNA binding activity. Nucleic acids res 30(21): 4607-4617.

2. Droll D, Archer S, Fenn K, Delhi P, Matthews K, et al. (2010) The trypanosome Pumilio-domain protein PUF7 associates with a nuclear cyclophilin and is involved in ribosomal RNA maturation. FEBS Lett 584(6): 1156-1162.

3. Fan Q Li J, Kariuki M, Cui L (2004) Characterization of PfPuf2, member of the Puf family RNA-binding proteins from the malaria parasite Plasmodium falciparum. DNA cell biol 23(11): 753-760.

4. Gomes-Santos CS, Braks J, Prudêncio M, Carret C, Gomes AR, et al. (2011) Transition of Plasmodium sporozoites into liver stage-like forms is regulated by the RNA binding protein Pumilio. PLoS Pathog 7(5): e1002046. 


\section{International Journal of Zoology and Animal Biology}

5. Hoek M, Zanders T, Cross GA (2002) Trypanosoma brucei expression-site-associated-gene-8 protein interacts with a Pumilio family protein. Mol Biochem Parasitol 120(2): 269-283.

6. Liang X, Hart KJ, Dong G, Siddiqui FA, Sebastian A, et al. (2018) Puf3 participates in ribosomal biogenesis in malaria parasites. J Cell Sci 131(6): jcs212597.

7. Lindner SE, Mikolajczak SA, Vaughan AM, Moon W, Joyce BR, et al. (2013) Perturbations of Plasmodium Puf2 expression and RNA-seq of Puf2-deficient sporozoites reveal a critical role in maintaining RNA homeostasis and parasite transmissibility. Cell Microbiol 15(7): 1266-1283.

8. Min Liu, Jun Miao, Tingkai Liu, William JS, Liwang C, et al. (2014) Characterization of TgPuf1, a member of the Puf family RNA-binding proteins from Toxoplasma gondii. Parasit Vectors 7: 141.

9. Miao J, Fan Q Parker D, Li X, Li J, et al. (2013) Puf mediates translation repression of transmissionblocking vaccine candidates in malaria parasites. PLoS Pathog 9(4): e1003268.

10. Miao J, Li J, Fan Q, Li X, Li X, et al. (2010) The Puffamily RNA-binding protein PfPuf2 regulates sexual development and sex differentiation in the malaria parasite Plasmodium falciparum. Journal of cell science 123(Pt 7): 1039-1049.
11. Müller K, Matuschewski K, Silvie O (2011) The Puffamily RNA-binding protein Puf2 controls sporozoite conversion to liver stages in the malaria parasite. PloS one 6(5): e19860.

12. Sony Shrestha, Xiaolian Li, Gang Ning, Jun Miao, Liwang Cui (2016). The RNA-binding protein PfPuf1 functions in the maintenance of gametocytes in Plasmodium falciparum. J Cell Sci 129(16): 31443152.

13. Tadauchi T, Matsumoto K, Herskowitz I, Irie K (2001) Post-transcriptional regulation through the HO 3'UTR by Mpt5, a yeast homolog of Pumilio and FBF. EMBO J 20(3): 552-561.

14. White EK, Moore Jarrett T, Ruley HE (2001) PUM2, a novel murine puf protein, and its consensus RNAbinding site. RNA 7(12): 1855-1866.

15. Zamore PD, Williamson JR, Lehmann R (1997) The Pumilio protein binds RNA through a conserved domain that defines a new class of RNA-binding proteins. RNA 3(12): 1421-1433.

16. Zhang B, Gallegos M, Puoti A, Durkin E, Fields S, et al. (1997) A conserved RNA-binding protein that regulates sexual fates in the $\mathrm{C}$. elegans hermaphrodite germ line. Nature 390(6659): 477-484.

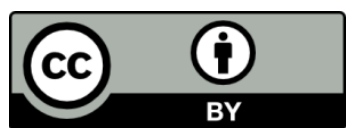

